Unfallchirurg 2014 • 117:755-755

DOI 10.1007/s00113-014-2597-0

Online publiziert: 15. August 2014

(c) Springer-Verlag Berlin Heidelberg 2014
B. Geiges ${ }^{1} \cdot$ C. von Falck ${ }^{2} \cdot$ K. Knobloch ${ }^{3} \cdot$ C. Haasper ${ }^{4} \cdot$ R. Meller ${ }^{4} \cdot$ C. Krettek ${ }^{4}$. S. Hankemeier ${ }^{4} \cdot$ J. Brand $^{5} \cdot$ M. Jagodzinski $^{4}$

${ }^{1}$ Abteilung Orthopädie/Unfallchirurgie/Sportmedizin, Agnes-Karll-Krankenhaus, Laatzen

${ }^{2}$ Zentrum für Radiologie, Medizinische Hochschule Hannover

${ }^{3}$ Klinik für Plastische, Hand- und Wiederherstellungschirurgie, Medizinische Hochschule Hannover

${ }^{4}$ Klinik für Unfallchirurgie, Medizinische Hochschule Hannover

${ }^{5}$ Praxisklinik und Chirurgische Praxis Brand, Dyck und Schulz, Uelzen

\section{Retraction Note to:}

Biodegradierbare Schraube

vs. einer Press-fit-Verankerung

für VKB-Rekonstruktionen

\section{Eine prospektive randomisierte Studie}

Der Artikel wurde von den Autoren zurückgezogen, da wesentliche Teile und Ergebnisse des Manuskripts bereits zuvor veröffentlicht wurden in:

This article has been retracted by the authors because substantial parts and results of the manuscript were previously published in:

Jagodzinski M, Geiges B, von Falck C, Knobloch K, Haasper C, Brand J, Hankemeier S, Krettek C, Meller R (2010) Biodegradable screw versus a press-fit bone plug fixation for hamstring anterior cruciate ligament reconstruction: a prospective randomized study. Am A Sports Med 38:501508. doi: 10.1177/0363546509350325. Epub 2009 Dec 31.

\section{Korrespondenzadresse}

\section{B. Geiges}

Abteilung Orthopädie/Unfallchirurgie/ Sportmedizin, Agnes-Karll-Krankenhaus Hildesheimer Str. 158, 30880 Laatzen bjoern.geiges@krh.eu 